\title{
PATRONES DE MARCACIÓN DE INGREDIENTES Y ORDEN DE PROCESOS EN TEXTOS GASTRONÓMICOS COSTARRICENSES
}

\author{
Patterns for marking ingredients and order of processes \\ in Costa Rican gastronomic texts
}

\author{
Mario Hernández Delgado* \\ Jorge Antonio Leoni de León ${ }^{* *}$ \\ Édgar Casasola Murillo***
}

\section{RESUMEN}

\begin{abstract}
En este artículo, se presentará una síntesis de los procedimientos y resultados relativos a la primera etapa, o etapa precomputacional, del proyecto "Análisis de contextos definicionales en corpus de gastronomía tradicional en Costa Rica (CODEGAT)”, en el que se estudian los recursos de marcación de informaciones gastronómicas presentes en corpus textuales en español en Costa Rica. Concretamente, se expondrán las tareas para la identificación de patrones asociados con dos tipos de rasgos necesarios para la configuración de una receta, que son "ingredientes" y "orden de procesos", para, finalmente, ofrecer una propuesta de categorización de los marcadores que aparecen en tales patrones, con ejemplos típicos de cada clase y de sus posibles contextos de aparición.
\end{abstract}

Palabras clave: lexicografía, lexicografía semiespecializada, patrones definicionales, marcadores definicionales, relaciones léxico-semánticas.

\begin{abstract}
In this paper, a synthesis of the procedures and results related to the first stage or pre-computational stage of the research project "Análisis de contextos definicionales en corpus de gastronomía tradicional en Costa Rica (CODEGAT)" ["Analysis of definitional contexts in corpora of traditional gastronomy in Costa Rica (CODEGAT)"] will be presented; in that stage, the linguistic resources for marking conceptual relations in gastronomic textual corpora in Costa Rican Spanish are studied. Specifically, the tasks for the identification of patterns associated with two types of features that were found as necessary in the formulation of a recipe ("ingredients" and "processes order") will be explained, to finally propose a categorization of the markers that appear in such patterns, with typical examples of each class and of its possible contexts of appearance.
\end{abstract}

Key Words: lexicography, semi-specialized lexicography, definitional patterns, definitional markers, lexical-semantic relations.

\footnotetext{
*Universidad de Costa Rica. Programa Estudios de Lexicografía. Costa Rica.Correo electrónico: pdfmario@gmail.com.

***Universidad de Costa Rica. Profesor de la Escuela de Filología, Lingüística y Literatura y Posgrado en Lingüística. Costa Rica. Correo electrónico: a.leoni@me.com.

***Universidad de Costa Rica. Profesor de la Escuela de Computación e Informática y Posgrado en Computación. Costa Rica. Correo electrónico: casasola@gmail.com.
} 


\section{Introducción}

Aunque no hay ningún tipo de necesidad lógica que imponga que la definición de un conjunto de unidades léxicas o léxico-conceptuales deba hacerse por medio de enunciados basados en lenguaje natural, ${ }^{1}$ lo cierto es que las formulaciones que emplean enunciados naturales (en oposición a los simbólicos y formalizados) pueden tener ciertas ventajas comunicativas: una de ellas es que tales enunciados pueden ser integrados eventualmente en la cadena discursiva de forma también natural; otra es que no requieren un entrenamiento especial para interpretarlos.

Por otro lado, si el conjunto de unidades léxicas que se define por medio de enunciados naturales es de cierta extensión, hay motivos que justifican el uso de algunas expresiones recurrentes en las formulaciones. Por ejemplo, desde el punto de vista de su elaboración (y aquí nos concentramos en las formas escritas), su empleo permite que el tiempo y esfuerzo del redactor se concentren en seleccionar y combinar las propiedades descriptivas pertinentes, en vez de intentar en cada definición ejercitar la variación estilística. Otra de las razones, esta vez desde el punto de vista del receptor, es que una forma lingüística recurrentemente asociada a contenidos análogos facilita la identificación de las relaciones semánticas del mismo tipo.

Ahora bien, el estudio de patrones y marcadores definicionales en español ha tenido su mayor impulso al interior de las investigaciones sobre contextos definitorios, pues aquellos constituyen la base que permitiría el reconocimiento y extracción automática de relaciones semánticas o conceptuales (véase, p. e., Sierra y Alarcón, 2002; Alarcón, 2003; Soler, 2005; Sierra, Alarcón y Aguilar, 2006; Aguilar, 2009; Sierra, 2009).

Sin embargo, el análisis de los marcadores lingüísticos (y eventualmente de otros tipos de recursos gráficos) empleados de manera sistemática y recurrente en enunciados definitorios puede también constituir una línea de investigación autónoma cuyos resultados descriptivos podrían servir a otros fines, como, por ejemplo, la estandarización de las definiciones de una misma obra, campo, disciplina, etc. (véase, p. e., Alcina y Valero,

\footnotetext{
${ }^{1}$ Baste considerar que las descripciones tabulares hechas con los procedimientos del análisis componencial, o las estructuraciones producidas por las ontologías formales, producen análogos efectos informativos y oposicionales.
} 
2008; Valero, 2009; Valero y Alcina, 2009). Esa expectativa queda clara en las siguientes palabras de Valero (2009):

Nuestro proyecto tiene como objetivo diseñar una herramienta de terminografía asistida que ayude al terminógrafo/a a elaborar las definiciones de conceptos especializados. Estas definiciones, basadas en patrones de definición, serán completas y coherentes con todo el sistema de definiciones y contendrán la información de manera estructurada. Uno de los elementos del patrón de definiciones consiste en un conjunto de marcadores lingüísticos introductorios de las diferentes características en la definición. Este análisis nos ha ayudado a observar los marcadores que ocurren de manera natural en el texto de las definiciones. En futuros trabajos nos planteamos seleccionar uno o varios de estos marcadores para cada característica e introducirlos en el patrón de este grupo conceptual (p. 11).

En lo que respecta al presente artículo, se describen algunos resultados parciales de una investigación enmarcada en la línea de estudio conocida como análisis de contextos definicionales (o definitorios); específicamente, los resultados relativos a la identificación de marcadores lingüísticos vinculados a patrones semánticos relevantes.

\section{El análisis de contextos definicionales}

El análisis de contextos definicionales consiste en el estudio de corpus textuales amplios y ricos en información sobre los significados, sentidos o usos de unidades léxicas o terminológicas relativas a áreas de conocimiento especializadas para identificar y extraer datos semánticos relevantes sobre tales unidades. Para efectos de su tratamiento teórico y metodológico, muchas veces a tales áreas de conocimiento especializadas, en especial cuando se manifiestan textualmente y contienen muchas informaciones definitorias de unidades terminológicas, o de unidades léxicas con cierto grado de especialización, se les conoce como dominios especializados o dominios restringidos.

Existen diversas disciplinas interesadas en la definición de unidades léxicas (palabras, términos, locuciones, expresiones), como la lexicografía, la terminología y la linguiística computacional, entre otras. Independientemente de si la posición teórica desde la que se 
investiga concibe que lo que se define son conceptos (que es una posición de índole psicológica-cognitiva), entidades referenciales (posición realista propia de la terminología, las tecnologías, las ciencias exactas y naturales e, incluso, del "sentido común”) o unidades léxicas (que es la posición lingüística-lexicográfica), para definir una unidad con contenido conceptual o semántico, especializada o no, se necesita tener información proveniente de la comunidad que utiliza tales unidades léxicas. Una de las fuentes más valiosas para extraer esos conocimientos socializados son los corpus textuales en los que se utilizan esas unidades. De hecho, el acceso a corpus textuales masivos es una de las ventajas de la revolución que ha significado el gran avance de las tecnologías de la información.

La principal diferencia entre el análisis de contextos definicionales y los procedimientos tradicionales por los que el lexicógrafo, el terminólogo u otros profesionales buscan y procesan las informaciones sobre el contenido de determinadas unidades léxicas o terminológicas, es que el primero se basa en el reconocimiento y estudio de patrones lingüísticos recurrentes para determinar la existencia, pertinencia y organización de bloques de informaciones definitorias en una masa textual.

Uno de los mayores beneficios de este tipo de estudios es que facilitan la tarea de clasificar y sistematizar las informaciones definicionales pertinentes a un dominio restringido particular. Eso a su vez permite, entre otras cosas, proponer formas estandarizadas para definir las palabras o términos de ese ámbito, con el objetivo de mejorar las formas de transmitir, compartir, comparar e identificar las relaciones conceptuales relevantes.

\section{La receta gastronómica como definición especializada}

En el caso del proyecto "Análisis de contextos definicionales en corpus de gastronomía tradicional en Costa Rica (CODEGAT)”, se estudian los recursos de marcación de informaciones gastronómicas presentes en corpus textuales en español de Costa Rica.

Visto lo culinario como una actividad de importancia social, estudios como este pueden ayudar a sistematizar el conocimiento que la comunidad posee sobre este campo de actividad, de modo que se pueda resguardar tal conocimiento mediante la identificación de 
las informaciones distintivas y notables tocantes a la preparación de recetas reconocidas como tradicionales en el ámbito costarricense.

Para efectos de este proyecto, se conceptualizó una receta culinaria como una definición de índole semiespecializada que describe el contenido conceptual asociado con el nombre de esa receta. Más específicamente, como una formulación linguiísticoconceptual de carácter procesual que describe, partiendo de la declaración de un conjunto de componentes (principalmente ingredientes), la forma de llegar desde un estado inicial en el que los componentes coexisten sin agregación entre ellos, a un estado final en que, de acuerdo con ciertos procesos aplicados en cierto orden lógico y temporal, se produce un resultado (culinario, en este caso). Ese resultado sería el que, una vez abstraídos la mayoría de los rasgos semánticos especializados, y manteniéndose solamente los diferenciadores, constituye el contenido semántico de la unidad léxica que da nombre a la receta para efectos de verse integrada en el sistema léxico de la lengua -es decir, el hablante generalmente no asocia el nombre del plato con todo el procedimiento ni con el conjunto exhaustivo de sus ingredientes, sino con ciertas características básicas y el producto final-.

El hecho de que aquí se piense la receta culinaria como una definición semiespecializada no significa que se considere como una construcción definitoria necesariamente hecha por especialistas ni dirigida necesariamente a un público con estudios formales en gastronomía o que se dedique profesionalmente a ello. Tampoco que esté confeccionada con bases teóricas o metodológicas rigurosas. Desde la perspectiva de este trabajo, "semiespecializada" significa que cumple dos características:

a) Que es adecuada en términos de distintividad (característica que concuerda con una definición lexicográfica "no especializada", lo cual se basa en el requisito, teórico o ideal, de distinguir en forma suficiente lo definido de otros elementos dentro de un sistema de oposiciones).

b) Que puede extenderse en informatividad tanto como se crea necesario para permitir la comprensión de lo definido (en lo cual difiere del "ideal teórico" de la definición lexicográfica, para el cual el exceso de información sería, en principio, un defecto).

En realidad, la lexicografía especializada no debería verse como si fuera un punto extremo del espectro de la actividad lexicográfica, sino como un continuum de 
posibilidades, o al menos como una serie de grados que parten de la definición/obra lexicográfica sin marca de especialidad y avanzan hacia la definición terminológica (sin llegar a esta, porque la terminología implica un salto categorial de carácter teórico y metodológico respecto de la lexicografía). Por lo tanto, al considerar una receta gastronómica como una definición semiespecializada, lo que se hace es ubicarla en algún punto del continuum (o de la sucesión graduada) que se ilustra en la Figura 1:

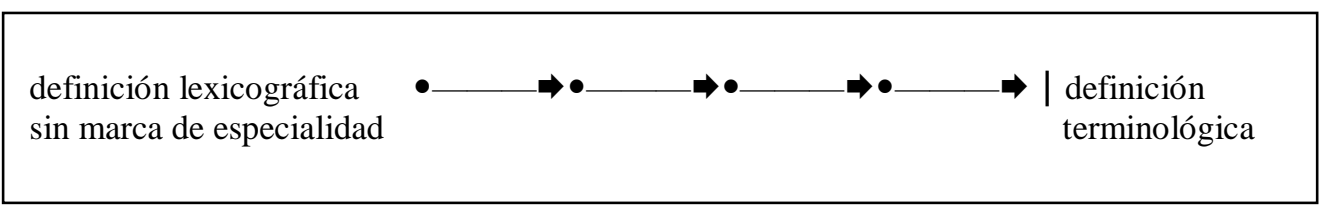

Figura 1. Eje de especialización de una definición

La principal diferencia entre la descripción de un plato tal como aparece definido en un diccionario (general, regional o con cualquier tipo de diarrestricción de uso) y la descripción de un plato tal como aparece en un recetario (aparte de las obvias convenciones, notaciones y simbologías propias de la obra lexicográfica formal), es que, en el diccionario, el nombre del plato es considerado como una unidad léxica que se encuentra en oposición a otras unidades léxicas del sistema lexicológico dado, y su definición lo que intenta hacer es describir los rasgos de contenido que oponen suficientemente (y no necesariamente de modo exhaustivo) su significado al de otras unidades léxicas del mismo sistema. Véanse los siguientes ejemplos (DEL, 2014):

$\operatorname{arroz}(\ldots)$

\section{a la chorrera.}

1. m. Cuba. Plato de pollo o gallina con arroz caldoso.

Aunque esta definición es omisa en aclarar si el pollo o gallina van revueltos con el arroz caldoso o si se sirven a la par, al menos es suficiente para saber que un "arroz a la chorrera" no es un tipo de sopa ni de ensalada ni de picadillo; y, además, que no es un arroz con pescado ni un arroz con vegetales ni un arroz con leche, etc.

lasaña (...). 
1. f. Plato formado por capas de pasta de harina, cuadradas o alargadas, que se intercalan con carne picada, verdura, etc.

En esa definición, no se indica la forma en que se procesan los componentes o el conjunto, ni los condimentos que llevaría o los tiempos de procesamiento - de hecho, así

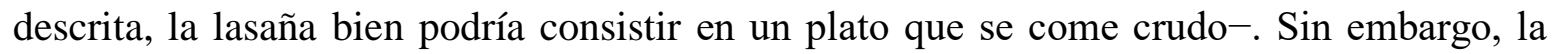
información que da es suficiente para diferenciar el plato llamado "lasaña" de cualquier otro que no se prepare con capas de pasta de harina o que las lleve, pero que estas no se intercalen con carne picada ni verduras, etc.

Mas considérese ahora la siguiente receta:

\section{SOPA DE ROPA VIEJA}

\section{Ingredientes:}

1 litro de leche

1 cuajada

1 pelota de masa

1 cebolla

1 chile dulce

2 ajos

sal

4 hojas de culantro de coyote

achiote

\section{Preparación:}

Se pone a hervir la leche con los olores.

Se mezcla la cuajada con la masa y se hacen las pelotitas y se ponen a freír. Cuando ya están listas se mezclan con la leche.

Sal al gusto, achiote al gusto y a disfrutar de una deliciosa y alimenticia sopa. 
En el caso de esa receta de la "sopa de ropa vieja", tomada de la compilación Cocina tradicional costarricense 1: Guanacaste y Región Central de Puntarenas, realizada por Álvarez Masís (2005), se observa las siguientes características:

a) El nombre del plato va seguido por una serie de informaciones que describen el conocimiento asociado a la confección de tal plato.

b) Hay al menos dos rasgos pertinentes asociados con la descripción (con la "receta"), los cuales se presentan separados en bloques bien diferenciados. Estos son: ingredientes y preparación.

c) Aunque no todos los pasos llevan una marca lingüística que indique el orden de unos respecto de otros, algunos sí lo tienen (“se... y se... y se...”, “cuando ya...”, “у a disfrutar de"). Debido a eso, se postula en este artículo que el rasgo "orden de procesos" es un tercer rasgo pertinente del contenido semántico de una receta. Además, se considera el rasgo "orden de procesos o de pasos" como un rasgo subordinado al rasgo "preparación", pues especifica y aclara las modalidades lógicas o temporales de este último. Aunque habría otros tipos de rasgos potencialmente pertinentes, como, por ejemplo, "utensilios" y "tiempos de procesamiento", la poca regularidad de los marcadores utilizados ha hecho que, al menos en esta etapa del proyecto, no sean considerados para el análisis.

Debido a la inspección de casos de formulaciones gastronómicas como la anterior, se considera que los rasgos "ingredientes" y "orden de procesos" son pertinentes y fundamentales para la concepción de una receta, así como para su descripción.

\section{Metodología}

En términos globales, la metodología de análisis por contextos definicionales tiene dos grandes etapas, que son:

a) Identificación de los marcadores empleados para señalar la presencia de informaciones definicionales (rasgos pertinentes), donde cada marcador particular, juntamente con el tipo de información conceptual con la que concurre, constituye un patrón. 
b) Aplicación de procedimientos de reconocimiento automático, en corpus masivos, de contextos informativos a partir de los marcadores propuestos, para verificar que, efectivamente, son funcionales como indicadores de rasgos pertinentes del dominio de especialidad estudiado.

La idea general se basa en la expectativa de contar con un conjunto de formas (generalmente lingüísticas, aunque podrían incluirse también otros tipos de recursos gráficos) que funcionen como "señalizadores" para ubicar relaciones conceptuales definicionalmente relevantes en un corpus textual masivo, incluso en uno indiferenciado o no estructurado.

En esta comunicación se presentan los procedimientos y ejemplos de los resultados relativos a la primera etapa, o etapa precomputacional. Esta investigación se encuentra aún en desarrollo, pero, en términos globales, el procedimiento seguido hasta el momento consiste en las siguientes actividades:

a) Constitución de un corpus textual fuente abundante en información definicional del campo culinario costarricense. Luego de una amplia búsqueda bibliográfica, se seleccionó un conjunto de cinco recetarios compilados por Álvarez Masís (2005, 2007, 2010, 2013a y 2013b), los cuales fueron publicados como serie y abarcan recetas de las siete provincias de Costa Rica.

b) Se obtuvo una muestra del $30 \%$ de ese corpus, a partir de la cual se realizó una inspección para determinar las informaciones relevantes y pertinentes que constituyen una receta y, como ya se mencionó, se detectó que ingredientes y orden de procesos son los rasgos de mayor regularidad y riqueza en cuanto a patrones de marcación, por lo que se decidió trabajar con ellos.

c) Se hizo una revisión "manual" (esto es, visual, no automatizada) en busca de patrones recurrentes asociados, correspondientemente, a ingredientes y a orden de procesos, identificando a la vez los candidatos a marcadores empleados para cada una de esas clases de rasgos.

d) Paralelamente, se buscó en internet un corpus fuente también rico en informaciones definicionales gastronómicas (i. e., recetas) y que fuera apto para realizar 
pruebas de reconocimiento para evaluar la funcionalidad de las formas propuestas como candidatas a marcadores según el corpus base. Se buscó y seleccionó un sitio web completo dedicado a la cocina costarricense y se extrajo todo el texto útil del sitio, manteniendo la estructura lineal; luego se depuró, normalizó y pasó a texto plano, constituyendo así un corpus inicial usado con carácter anónimo para hacer pruebas piloto de los procedimientos (CP).

e) Sobre ese corpus CP se realizaron pruebas piloto para la identificación de contextos definicionales de ingredientes a partir de los marcadores propuestos.

f) Se ha realizado la inspección del corpus base para identificar candidatos a marcadores de orden de procesos. Debido a que en este caso hay más variedad y complejidad que para los de ingredientes, se han requerido varias "pasadas" y afinamientos. La evaluación de funcionalidad en el corpus de pruebas está aún pendiente.

\section{Resultados}

\subsection{Ingredientes}

En forma concreta, los resultados principales son los siguientes:

a) Existen casos de ingredientes no asociados con una forma lingüística o gráfica que sea funcional como marcador, como en el caso de "sal" y "achiote" en la receta de la "sopa de ropa vieja" anteriormente presentada. Estos casos no son directamente útiles para el tratamiento por análisis de contextos definitorios.

b) Los marcadores son siempre "operacionalmente sincréticos", en el sentido de que en cada uno de ellos siempre convergen al menos dos tipos de informaciones útiles para efectos de la identificación de ingredientes, que son: i) señalamiento del punto dentro del bloque textual donde se declara cada uno de los ingredientes asociados a marcadores; y ii) modalidad del ingrediente, como cantidad exacta (5, una cucharada, dos latas, 1 kilo), cantidad aproximada (una pizca, un poquito), cantidad subjetiva (al gusto, lo que se quiera), forma de presentación (en juliana, picados, machacado, ralladas) u otras. 
c) Las categorías de marcación de ingredientes que se han detectado como pertinentes en la declaración de ingredientes (y de las cuales quien redacta la receta escoge las que considere convenientes) se indican en la Tabla 1. Para cada una, se dan ejemplos representativos generalizados, mas no la lista exhaustiva, pues esta todavía requiere más pruebas de procesamiento computacional para validar su funcionalidad: ${ }^{2}$

\section{Tabla 1}

Marcadores de ingredientes

\begin{tabular}{|c|c|c|}
\hline Categoría & Ejemplos de marcadores & Ejemplos contextuales \\
\hline \multirow[t]{3}{*}{ Cantidad numérica exacta } & _\#_ & 3 ajos \\
\hline & _\#\#_ & 15 chayotes \\
\hline & \#/\#_ & $1 / 2$ cebolla \\
\hline \multirow{5}{*}{$\begin{array}{l}\text { Cant. por } \\
\text { convencional }\end{array}$} & \#_taza(s)_de_ & 1 taza de aceite \\
\hline & \#_kilo(s)_de_ & 2 kilos de espinacas \\
\hline & \#_sobre(s)_de_ & 1 sobre de consomé \\
\hline & \#_cucharad(it)a(s)_de_ & 2 cucharadas de aceite \\
\hline & \#/\#_rollo_de_ & $1 / 2$ rollo de orégano \\
\hline \multirow[t]{2}{*}{ Cantidad por unidad natural } & \#_rama(s)_[[X_]]de_ & 1 rama pequeña de apio \\
\hline & \#_hojas_de_ & 4 hojas de laurel \\
\hline
\end{tabular}

${ }^{2}$ En el caso de los marcadores de ingredientes, se omitieron las definiciones de las categorías debido a que se pueden inferir fácilmente del nombre de cada una junto con los marcadores-tipo con que se ilustran y sus ejemplos contextuales. 


\begin{tabular}{lll}
\hline Cantidad aproximada & 1_poquito_de_ & 1 poquito de achiote \\
\hline una_pizca_de_ & una pizca de sal \\
\hline un_poco_de_ & un poco de salsa lizano o similar \\
\hline 1_pedazo_de_ & 1 pedazo de apio \\
\hline Cantidad subjetiva & _al_gusto & aceite al gusto \\
\hline (al_gusto) & ajinomoto (al gusto) \\
\hline Cantidad indefinida & el/la(s)/lo(s)_necesario/a(s)_[[para & azúcar en polvo (la necesaria para \\
& d] & decorar) \\
\hline & el/la(s)/lo(s)_suficiente(s)_[[para] & agua la suficiente
\end{tabular}

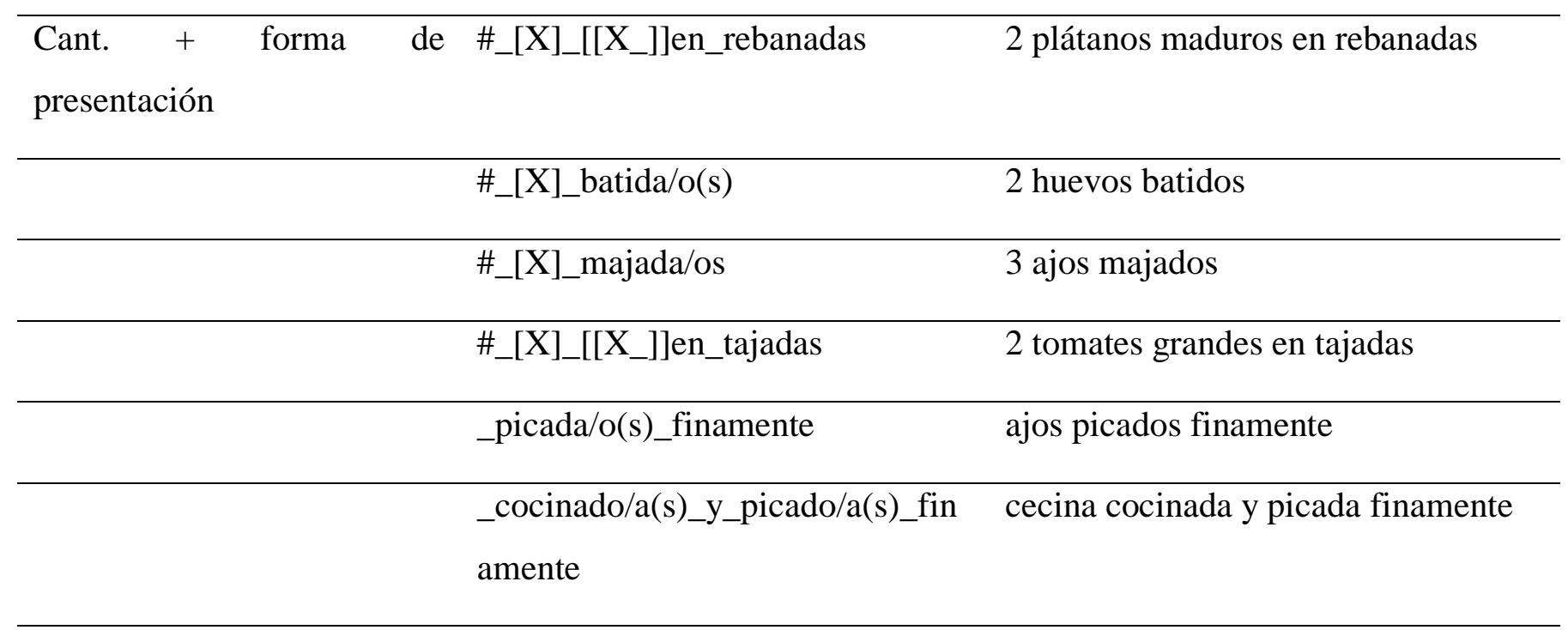




\begin{tabular}{|c|c|c|}
\hline Cantidad + estado & \#_[X]_tierna/o(s) & 2 chayotes tiernos \\
\hline & \#_[X]_cruda/o(s) & 4 huevos crudos \\
\hline & _en_rama & culantro en rama \\
\hline \multirow[t]{2}{*}{ Cantidad + tamaño } & \#_[X]_grande(s) & 2 cebollas grandes \\
\hline & \#_[X]_pequeña/o(s)[[_]] & 1 cebolla pequeña bien picada \\
\hline \multirow[t]{4}{*}{ Opcionalidad } & si_[[se_]]desea_agregue_ & Si se desea agregue achiote \\
\hline & _(opcional) & chile picante (opcional) \\
\hline & ,_opcional & salsa inglesa, opcional \\
\hline & opcional:_ & $\begin{array}{l}\text { Opcional: chile picante, orejas y rabo } \\
\text { de cerdo }\end{array}$ \\
\hline
\end{tabular}

\subsection{Orden de procesos}

De forma igualmente sintética, las principales observaciones son:

a) Del mismo modo que como ocurre con los ingredientes, se constató que no siempre se utilizan cadenas de caracteres de interpretación lingüística que se puedan postular como candidatos a marcadores. Por ejemplo, existen casos, como en el ejemplo de la "sopa de ropa vieja" (expuesto antes y cuya sección de "preparación" se reproduce abajo), en que algunas relaciones conceptuales de ordenación de procesos se señalan por medio de convenciones no propiamente lingüísticas, sino con el uso de la puntuación o el cambio de línea. Si bien ese tipo de recursos son generalmente suficientes para motivar que se interprete la yuxtaposición como secuenciación lógica o temporal (como en el paso de la instrucción 1 a la 2, de la 5 a la 6 y de esta última a la 7), no son aptos para su tratamiento por medio de análisis de contextos definitorios. ${ }^{3,4}$

\footnotetext{
${ }^{3}$ La indicación 8 no es en realidad un paso ni una instrucción de la receta, sino más bien un patrón de cierre, donde el marcador de finalización correspondiente sería "a disfrutar de" o "y a disfrutar de".
} 


\section{Preparación:}

[1] Se pone a hervir la leche con los olores.

[2] Se mezcla la cuajada con la masa y [3] se hacen las pelotitas y [4] se ponen

a freír. Cuando ya están listas [5] se mezclan con la leche.

[6] Sal al gusto, [7] achiote al gusto y [8] a disfrutar de una deliciosa y alimenticia sopa (Álvarez Masís, 2005, la numeración entre corchetes no es del original).

b) Igual que ocurre con los marcadores de ingredientes, los que indican ordenación de los procesos también cumplen simultáneamente dos funciones desde una perspectiva operativa: i) señalan la presencia de un paso, proceso o momento dentro del procedimiento global de la receta, diferenciándolo del resto; y ii) indican las relaciones conceptuales (generalmente de índole lógica o temporal) entre los diferentes pasos. Puede haber, por ejemplo, marcadores de inicio (primero, para comenzar), de finalización (por último, y listo para servir, ¡listo!), fin de paso (hasta que), simultaneidad (mientras tanto), diversos tipos de secuencialidad (después, cuando ya, de nuevo, la noche antes), duración del proceso (por un rato) u otros.

c) A continuación se indican las categorías en que se han agrupado los diferentes marcadores de orden de procesos, cada una con su respectiva caracterización y, más abajo (Tabla 2), ejemplos de marcadores representativos de cada una de ellas, junto con posibles contextos de aparición -vale también aquí lo dicho para el caso de las categorías de marcadores de ingredientes, en el sentido de que la generalización de las formas, así como las categorías mismas, son susceptibles de afinamiento de acuerdo con lo que sugieran las pruebas de recuperación automatizada-:

\footnotetext{
${ }^{4}$ Un análisis por medio de explicaturas podría intentar reconstruir la estructura interproposicional, pero ese tipo de enfoque pragmático-cognitivo no es, al menos por el momento, compatible con un análisis de contextos definicionales, que se basa en indicadores efectivamente codificados.
} 
Marcador de inicio: indica el comienzo de la receta.

Marcador de finalización: indica la conclusión de la receta.

Marcador de fin de paso: señala el momento en que un paso se puede dar por concluido al haberse logrado el estado deseado de aquello a lo que se aplicaba la acción.

Marcador de simultaneidad: indica que determinado paso se realiza al mismo tiempo que otro.

Marcador de secuencialidad simple: indica secuencialidad entre pasos, ya sea de forma explícita o porque permite inferirla.

Marcador de secuencialidad compleja: señala la concatenación secuencial de al menos tres pasos o procesos.

Marcador de secuencia diferida: señala o sugiere un amplio lapso entre un paso y otro que se daría antes o después.

Marcador de secuencialidad por reiteración: indica que se hace algo idéntico o similar a otra cosa que ya se había hecho.

Marcador de secuencialidad inversa: implica deshacer, revertir, invertir o reintegrar a una situación original (generalmente una posición o estado) algo que se había puesto en determinado estado. El estado previo se infiere del señalado por el marcador.

Duración: indica la duración esperada o sugerida para un proceso o acción. 


\section{Tabla 2}

\section{Marcadores de orden de procesos}

\begin{tabular}{|c|c|c|}
\hline Categoría & Ejemplos de marcadores & Ejemplos contextuales \\
\hline \multirow[t]{3}{*}{ Inicio } & 1. & 1. Se lava bien el mondongo \\
\hline & Primero & Primero se lava el arracache \\
\hline & Comenzamos por & Comenzamos por rallar la zanahoria \\
\hline \multirow[t]{5}{*}{ Finalización } & i[X]! & ¡Luego a servir! \\
\hline & al_horno_a & al horno a dorar \\
\hline & _listo/a_para_servir & listo para servir \\
\hline & por último & por último se le añade el culantro \\
\hline & y_servir & y servir bien caliente sobre la carne \\
\hline \multirow[t]{4}{*}{ Fin de paso } & hasta_alcanzar & hasta alcanzar el punto de nieve \\
\hline & hasta_cristalizar & hasta cristalizar la cebolla \\
\hline & hasta_quedar & hasta quedar bien seca \\
\hline & hasta_que_[[se_]]dore(n) & hasta que se doren bien \\
\hline \multirow[t]{3}{*}{ Simultaneidad } & Aparte, & Aparte, en una olla \\
\hline & _por_aparte & $\begin{array}{l}\text { cocine por aparte el guineo o } \\
\text { plátano }\end{array}$ \\
\hline & _mientras_ & mientras en una sartén \\
\hline
\end{tabular}




\begin{tabular}{|c|c|c|}
\hline Secuencialidad simple & después_le & $\begin{array}{l}\text { después le agrega la leche } \\
\text { evaporada }\end{array}$ \\
\hline & después_a & después a freír \\
\hline & después_["verbo"] & después lava el arroz \\
\hline & luego_["verbo"] & luego echar los garbanzos \\
\hline & luego_se & luego se agregan los guineos \\
\hline & cuando_ya_ & cuando ya esté hirviendo (...) \\
\hline Secuencialidad compleja & se_[X]_y_se_[X]_y_se_[X] & $\begin{array}{l}\text { se mezcla la cuajada con la masa y } \\
\text { se hacen las pelotitas y se ponen a } \\
\text { freír. }\end{array}$ \\
\hline \multirow[t]{2}{*}{ Secuencialidad diferida } & _al_día_siguiente & al día siguiente se pica fina \\
\hline & _el_día_antes & se deja el día antes en refrigeración \\
\hline \multirow[t]{4}{*}{ Secuencialidad por reiteración } & _de_nuevo_ & $\begin{array}{l}\text { de nuevo al fuego para que reviente } \\
\text { bien }\end{array}$ \\
\hline & nuevamente & echar nuevamente olores \\
\hline & otra vez & otra vez al fuego \\
\hline & repita_la_operación & repita la operación \\
\hline \multirow[t]{2}{*}{ Secuencialidad inversa } & quite_del & quite del fuego \\
\hline & retirar(lo/a)(s)_del & retirarlo del fuego \\
\hline
\end{tabular}




\begin{tabular}{lll}
\hline Duración & durante_ & durante 45 minutos \\
\hline & por_\#(\#)_ & por 30 minutos \\
\hline${ }^{\prime}$ un_rat(it)o & un rato y se bota el agua \\
\hline$-\#(\#) \_$minuto(s) & Hervir durante 8 minutos \\
\hline$\_\#(\#) \_$hora(s) & dejarlo una hora en un poco de agua \\
\hline
\end{tabular}

\section{Conclusión}

Las tareas llevadas a cabo hasta el momento en cuanto a los rasgos "ingredientes" y "orden de procesos" en el corpus de trabajo seleccionado son, en resumen, las siguientes:

a) Identificación de patrones de inserción de ambos tipos de informaciones.

b) Identificación de configuraciones gráficas o lingüísticas concurrentes con las relaciones semánticas relativas a los diversos patrones.

c) "Aislamiento" de las formas o secuencias postuladas como "buenos" marcadores para el tipo de relación semántica respectiva.

d) Generalización de las formas respectivas, para ampliar el potencial alcance de cada clase de marcadores propuestos.

e) Clasificación de los marcadores según las diversas modalidades de los rasgos a los que cada uno señala.

f) Conceptualización y caracterización de cada categoría.

Tal como se indicó, los marcadores propuestos en esta etapa deberán ser puestos a prueba por medio de procedimientos automatizados para evaluar su efectividad como señalizadores de los tipos de informaciones respectivos, de modo que puedan servir para la recuperación automática de informaciones a partir de corpus textuales masivos, indiferenciados y no estructurados. Sin embargo, las categorías descritas, y las formas generalizadas propuestas, en esta misma etapa ya podrían servir como base para la propuesta de clases paradigmáticas de marcadores para una eventual estandarización de recetas en lo tocante a ingredientes y orden de procesos. 


\section{Bibliografía}

Aguilar, César. (2009). Análisis lingüístico de definiciones en contextos definitorios (Tesis doctoral). Universidad Nacional Autónoma de México.

Alarcón, Rodrigo. (2003). Análisis lingüístico de contextos definitorios en textos de especialidad (Tesis de licenciatura). Universidad Nacional Autónoma de México.

Alcina, Amparo y Valero, Esperanza. (2008). Análisis de las definiciones del diccionario cerámico científico-práctico. Sugerencias para la elaboración de patrones de definición. Debate Terminológico, 4. Recuperado de http://seer.ufrgs.br/index.php/riterm/article/download/23841/13830

Álvarez Masís, Yanory (Ed.). (2005). Cocina tradicional costarricense 1: Guanacaste y Región Central de Puntarenas. San José, Costa Rica: Ministerio de Cultura, Juventud y Deportes. Centro de Investigación y Conservación del Patrimonio Cultural: Imprenta Nacional.

Álvarez Masís, Yanory (Ed.). (2007). Cocina tradicional costarricense 2: Heredia y Limón. San José, Costa Rica: Ministerio de Cultura y Juventud. Centro de Investigación y Conservación del Patrimonio Cultural: Imprenta Nacional.

Álvarez Masís, Yanory (Ed.). (2010). Cocina tradicional costarricense 3: Cartago. San José, Costa Rica: Ministerio de Cultura y Juventud. Centro de Investigación y Conservación del Patrimonio Cultural: Imprenta Nacional.

Álvarez Masís, Yanory (Ed.). (2013a). Cocina tradicional costarricense 4: Alajuela y Heredia. San José, Costa Rica: Ministerio de Cultura y Juventud. Centro de Investigación y Conservación del Patrimonio Cultural/Instituto Costarricense de Turismo: Publiart, S. A.

Álvarez Masís, Yanory (Ed.). (2013b). Cocina tradicional costarricense 5: San José. San José, Costa Rica: Ministerio de Cultura y Juventud. Centro de Investigación y Conservación del Patrimonio Cultural/Instituto Costarricense de Turismo: Publiart, S. A. 
Real Academia Española. (2014). Diccionario de la Lengua Española (23. ${ }^{\mathrm{a}}$ ed.) [DLE]. Madrid: Espasa.

Sierra, Gerardo y Alarcón, Rodrigo. (2002). Identification of recurrent patterns to extract definitory contexts. Lecture Notes in Computer Science, 2276, 436-438.

Sierra, Gerardo, Pozzi, Mara y Torres, Juan Manuel (Eds.). (2009). Proceedings. (1. st) International Workshop on Definition Extraction, 18 de setiembre de 2009, Borovets, Bulgaria. Recuperado de https://aclweb.org/anthology/W/W09/W094400.pdf

Sierra, Gerardo, Alarcón, Rodrigo y Aguilar, César. (2006). Extracción automática de contextos definitorios en textos especializados. Revista de Procesamiento de Lenguaje Natural, 37, 351-352.

Sierra, Gerardo. (2009). Extracción de contextos definitorios en textos de especialidad a partir del reconocimiento de patrones lingüísticos. linguaMATICA, 2, 13-37.

Soler, Victoria. (2005). Patrones lingüísticos para la búsqueda de información conceptual en el corpus textual especializado de la cerámica TXTCera. Recuperado de http://repositori.uji.es/xmlui/bitstream/handle/10234/79115/forum_2004_50.pdf?seq uence $=1$

Valero, Esperanza y Alcina, Amparo. (2009). Linguistic realization of conceptual features in terminographic dictionary definitions. En Gerardo Sierra, Mara Pozzi y Juan Manuel Torres (Eds.), Proceedings. (1. ${ }^{\text {st }}$ ) International Workshop on Definition Extraction (54-60). Borovets, Bulgaria.

Valero, Esperanza. (2009). Los marcadores lingüísticos en las definiciones del grupo conceptual 'procesos de fabricación cerámica'. Recuperado de http://repositori.uji.es/xmlui/bitstream/handle/10234/78051/forum_2008_22.pdf?seq $\underline{\text { uence }=1}$

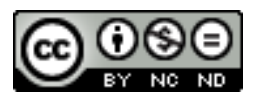

Esta obra está bajo una licencia de Creative Commons Reconocimiento-NoComercialSinObraDerivada 4.0 Internacional 